D) Check for updates

Cite this: Analyst, 2020, 145, 3634

Received 23rd September 2019,

Accepted 2nd January 2020

DOI: 10.1039/c9an01893j

rsc.li/analyst

\section{Determination of the concentration range for 267 proteins from 21 lots of commercial human plasma using highly multiplexed multiple reaction monitoring mass spectrometry $\dagger$}

\author{
Claudia Gaither, $\star^{\mathrm{a}}$ Robert Popp, $\ddagger^{\mathrm{a}}$ Yassene Mohammed (D) ${ }^{\mathrm{b}, \mathrm{c}}$ and \\ Christoph $\mathrm{H}$. Borchers (iD *b,d,e,f,g
}

\begin{abstract}
Multiple reaction monitoring (MRM) is a key tool for biomarker validation and the translation of potential biomarkers into the clinic. To demonstrate the applicability of MRM towards achieving this goal, we set out to determine the concentration ranges of 267 plasma proteins, including 61 FDA-approved/LDT developed biomarkers, in 21 commercial human plasma lots, as well as to assess accuracy and precision. Each target protein was quantified by calculating the area ratio of the endogenous tryptic target peptide to its stable isotope-labelled internal standard equivalent and compared to a standard curve. This highly multiplexed approach utilized a standard-flow UHPLC system linked to a triple quadrupole. All samples were analyzed across three separate days and assessed for robustness and accuracy. The standard curves and quality control samples showed excellent performance, with $>93 \%$ of standards and QCs meeting the acceptance criteria. A total of 248 proteins were able to be quantified in at least one sample on at least one of the three days, with 111 proteins being quantified in all 21 samples on all three days. The protein concentrations across all proteins covered six orders of magnitude. Furthermore, excellent three-day precision was demonstrated with $86 \%$ of CVs falling below $15 \%$. Overall, the protein concentration differences ranged from 1.1-fold for metalloproteinase inhibitor 2, to 69-fold for serum amyloid A-1/A-2.
\end{abstract}

\section{Introduction}

Over the past few decades, mass spectrometry-based proteomics has become an invaluable tool for unraveling the molecular blueprint of biology and disease, and to begin to understand their underlying complexity. Bottom-up discovery proteo-

\footnotetext{
${ }^{a}$ MRM Proteomics Inc., 141 Avenue du Président-Kennedy, SB-5100, C.P. \#26, Montreal, QC H2X 3X8, Canada

${ }^{b}$ University of Victoria - Genome British Columbia Proteomics Centre, University of Victoria, Victoria, British Columbia V8Z 7X8, Canada

${ }^{c}$ Center for Proteomics and Metabolomics, Leiden University Medical Center, Albinusdreef 2, Leiden, 2333 ZA, The Netherlands

${ }^{d}$ Department of Biochemistry and Microbiology, University of Victoria, Victoria, British Columbia, V8P 5C2, Canada

${ }^{e}$ Segal Cancer Proteomics Centre, Lady Davis Institute, Jewish General Hospital, McGill University, Montreal, Quebec, H3 T 1E2, Canada.

E-mail: christoph.borchers@mcgill.ca

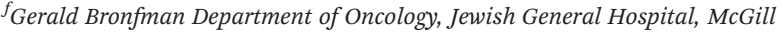
University, Montreal, Quebec, H3 T 1E2, Canada

${ }^{g}$ Department of Data Intensive Science and Engineering, Skolkovo Institute of Science and Technology, Skolkovo Innovation Center, Nobel St., Moscow 143026, Russia $\dagger$ Electronic supplementary information (ESI) available. See DOI: 10.1039/ c9an01893j

\$These authors contributed equally.
}

mics approaches using high resolution mass spectrometers in an untargeted fashion are commonly used to assess diseaseassociated proteins for up- or down-regulation, or for modifications such as post-translational modifications (PTMs). ${ }^{1}$ The identified proteins have the potential to be used as biomarkers for a variety of applications - including disease prognosis, diagnosis, and status, as companion diagnostics to determine eligibility for certain treatments, or for drug monitoring. After a potential biomarker or set of potential biomarkers has been identified, the biomarker candidates are transferred to a clinically compatible assay platform, followed by analytical and clinical validation. This transfer is required to overcome the limitations of the discovery platforms which include difficulty in standardizing measurements, the complexity of the workflow and data analysis which requires significant expertise, and low sample throughput.

The most common methodologies for biomarker validation and routine diagnostic use in clinical laboratories are the enzyme-linked immunosorbent assay (ELISA) and immunohistochemistry (IHC). ${ }^{2}$

Although robust, sensitive, amenable to automation, relatively easy to implement and perform once developed and validated, ELISAs rely heavily on the specificity of the antibody. 
Cross-reactivity of the antibodies with non-target antigens can result in protein overestimation and antigen recognition can be affected by unexpected or unknown PTM patterns that can result in an underestimation of protein expression. ${ }^{3}$ Moreover, ELISAs are susceptible to the Hook effect, which results in the underestimation of protein concentrations in samples with high protein concentrations. ${ }^{4}$ In addition, interferences by auto-antibodies have been reported. ${ }^{5}$

A powerful mass spectrometry-based technique, very suitable for studies designed to validate protein biomarkers, is multiple reaction monitoring (MRM), also called selected reaction monitoring (SRM). This is due to its high multiplexing capability (i.e., 10s to hundreds of proteins per run), its accuracy and precision when using stable isotope-labelled standard (SIS) peptides, its linear responses over several orders of magnitude, as well as low sample consumption. Furthermore, MRM achieves nearly absolute specificity due to the combination of peptide-specific retention time, fragment ion ratios, and mass-to-charge $(\mathrm{m} / \mathrm{z})$ information. ${ }^{6,7}$

MRM has been used for protein quantitation in various sample backgrounds, including human plasma, ${ }^{8}$ mouse plasma and tissues, ${ }^{9,10}$ and dried blood spots, ${ }^{11}$ among others. In addition to targeting non-modified peptides, MRM has been successfully applied to quantifying modified peptides either directly, e.g. phosphorylated $^{12}$ or glycosylated ${ }^{13}$ peptides, or after enzymatic removal of these modifications. ${ }^{14,15}$ Moreover, the enrichment of peptides prior to MRM analysis (immuno-MRM) can be used to increase sensitivity, if necessary. ${ }^{16,17}$

An important requirement for biomarker validation studies is the ability to achieve reproducible results between sample batches and between laboratories, and this has been demonstrated in many multi-laboratory studies using MRM. ${ }^{18-20}$ The use of standardized procedures and reagents, however, is required to achieve high reproducibility, which highlights the importance of commercially available reagent kits so that the research community can achieve transferable results. Our goal, therefore, was to address the need for standardization by developing easy-to-use MRM assay kits ${ }^{21}$ which had been rigorously validated following the Clinical Proteomic Tumor Analysis Consortium (CPTAC) guidelines. ${ }^{22}$

To expand upon previous work which resulted in a MRM panel for the quantitation of 76 human plasma proteins, ${ }^{21}$ the University of Victoria - Genome BC Proteomics Centre increased the number of protein targets to 267. Each of the 267 target proteins is quantified based on a unique tryptic peptide utilizing synthetic light and isotope-labelled standard peptides. The proteins cover a wide range of potential disease biomarkers, including neurodegenerative disease, renal disease, cancer, eye disease, bone disease, blood disorders, pregnancy complications, diabetes and autoimmune disorders among others. Of the 267 protein targets, 61 are FDA-approved protein biomarkers $^{23}$ and 67 are putative biomarkers for cardiovascular disease. ${ }^{24}$ Upon rigorous assay validation and interference screening following CPTAC guidelines, the MRM assays were turned into a kit and used for this study.
The kit consists of two lyophilized peptide mixtures, preweighed trypsin, and bovine serum albumin (BSA), as well as information on retention times, transitions, and optimized MS parameters for the following instruments: Agilent 6490/6495, Sciex 6500 Q-TRAP, and Thermo Q-Exactive. The first peptide mixture consists of 267 light (natural isotopic abundance, NAT) peptides that had been characterized for purity and concentration by capillary zone electrophoresis (CZE) and amino acid analysis (AAA). These peptides have been extensively screened for interferences during assay validation and are used to generate calibration curves with digested BSA as the background matrix. BSA background as a surrogate matrix for the generation of calibration curves has previously been shown to result in comparable accuracies as calibration curves generated in plasma. ${ }^{25}$ The second peptide mixture consists of the analogous 267 SIS peptides. These are spiked into both the plasma samples and the samples used to generate the calibration curve, and act as normalizers for ionization efficiency differences as well as any chromatographic variations between samples.

Here we show the human plasma panel performance as determined by quantifying target proteins from 21 lots of commercial human plasma from BioIVT (formerly known as Bioreclamation IVT).

\section{Experimental}

\section{Materials}

Reagents and labware. Phosphatase buffered saline (PBS) tablets, trizma pre-set crystals ( $\mathrm{pH} 8.0$ ), urea, dithiothreitol (DTT), and iodoacetamide (IAA) were purchased from Sigma Aldrich. Deep-well plates $(1.1 \mathrm{~mL})$ were purchased from AXYGEN. Protein LoBind tubes and LoBind 96-well PCR plates were purchased from Eppendorf. Oasis HLB $\mu$ Elution plates ( $2 \mathrm{mg}$ sorbent per well, $30 \mu \mathrm{m}$ particle size) were purchased from Waters. Ultrapure water was obtained with a Milli-Q Direct 8 water purification system. Formic acid (FA), methanol (MeOH) and acetonitrile (ACN) were purchased from Fisher Scientific. Eppendorf protein LoBind tubes were used for preparing the serial dilutions of the NAT mixture, and Falcon $15 \mathrm{~mL}$ conical tubes (Corning) were used for the preparation of the SIS mixture.

PeptiQuant 267-protein human plasma MRM panel. MRM Proteomics Inc.'s PeptiQuant ${ }^{\mathrm{TM}}$ 267-protein human plasma MRM assay kits contain light and heavy peptide mixes, as well as trypsin and BSA. The synthetic proteotypic peptides contained in the two mixtures (sequences shown in ESI Table $1 \dagger$ ) serve as molecular surrogates for the 267 human plasma proteins as described previously, following strict rules and criteria. ${ }^{26}$ Each protein is quantified by a single tryptic peptide to maximize the number of proteins quantifiable in a single run. The peptides were carefully selected using PeptidePicker ${ }^{27}$ to ensure protein-specific uniqueness and lack of modifications based on The Universal Protein Resource (UniProt). ${ }^{28}$ In cases where peptide variants have been documented within their sequences, the canonical sequence was selected unless specified. Similarly, when protein isoforms were noted, peptide 
sequences present in all isoforms were preferentially selected. When no peptide sequence present in all isoforms was found to meet all criteria, a peptide sequence found in the majority of isoforms was picked and the isoforms noted. Proteotypic peptides found in more than one plasma protein are noted. While the best possible peptides were selected for each protein, it should be kept in mind that nonetheless, in rare cases, gene mutations and/or PTMs could affect the trypsin cleavage efficiency. Each of the peptides was characterized for purity and accurate concentration by CZE and AAA, respectively. Furthermore, the synthetic peptides were tested for detectability when spiked into human plasma, and the ionization conditions were optimized empirically. Peptides were validated for use in LC/MRM-MS experiments, including establishing the limit of detection, linear range (lower limit of quantitation - LLOQ, and upper limit of quantitation - ULOQ), precision, and interferences, all in accordance with the CPTAC guidelines for assay development, ${ }^{22}$ which are available on the CPTAC assay portal website. ${ }^{29}$

Human plasma. Commercially available human plasma samples from 21 different lots were purchased from BioIVT. All the lots of human plasma analyzed were from whole blood donations. As shown in ESI Table $2, \uparrow$ ten of the samples were obtained from individual female donors, ten were from individual male donors, and one, BRH1447353, was a pooled human plasma sample. None of the samples had any indication of the health or disease state of the subjects other than testing negative for HIV $1 / 2 \mathrm{AB}$ and $\mathrm{HCV} \mathrm{AB}$, and being nonreactive for HBSAG, HIV-1 RNA, HCV RNA, HBV DNA, and STS, all in accordance with FDA regulations.

Repeatability assessment. The 21 human plasma samples were thawed once and divided into three aliquots, followed by storage at $-80{ }^{\circ} \mathrm{C}$ until the day of analysis. The samples were prepared on three different days with their own calibration curves and quality control samples (QCs) to determine the repeatability of the approach.

Digestion of human plasma and BSA surrogate matrix. The 21 different plasma lots and the BSA surrogate matrix were proteolytically cleaved with trypsin. Briefly, $10 \mu \mathrm{L}$ of either BSA at $10 \mathrm{mg} \mathrm{mL}^{-1}$ or human plasma were denatured and reduced at $\mathrm{pH} 8$ by addition of a urea/DTT/TrisHCl buffer at final concentrations of $6 \mathrm{M}$ urea, $13 \mathrm{mM}$ DTT, and $200 \mathrm{mM}$ TrisHCl, followed by incubation at $37^{\circ} \mathrm{C}$ for $30 \mathrm{~min}$. After denaturation and reduction, proteins were alkylated by adding IAA to a final concentration of $40 \mathrm{mM}$ and incubating at room temperature (RT) in the dark for $30 \mathrm{~min}$. After the alkylation step, TPCKtreated trypsin (Worthington) was added at a $20: 1$ (protein to enzyme, w/w) ratio, and samples were incubated overnight (18 hours) at $37{ }^{\circ} \mathrm{C}$ for proteolytic cleavage. The next day, the samples were acidified to a final concentration of $1.0 \% \mathrm{FA}(\mathrm{pH}$ $\leq 2$ ) to quench the digestion reaction, leading to a final peptide mixture with an estimated concentration of $1 \mu \mathrm{g} \mu \mathrm{L}^{-1}$. Samples were kept on ice until analyzed on the same day.

Reference standard and QC preparation. A digested $10 \mathrm{mg} \mathrm{mL} \mathrm{mL}^{-1}$ BSA-in-PBS-buffer surrogate matrix was used to prepare standards and QC samples. The lyophilized NAT peptide mix, previously balanced to the LLOQ of each peptide, was dissolved in $260 \mu \mathrm{L}$ of $30 \% \mathrm{ACN} / 0.1 \% \mathrm{FA}$ to give a final concentration of $100 \times$ LLOQ per $\mu \mathrm{L}$. This NAT peptide mixture was serially diluted with $30 \%$ ACN/0.1\%FA to yield eight concentrations: $100 \times, 40 \times, 16 \times, 4 \times, 2 \times, 0.5 \times, 0.25 \times$ and $0.1 \times$ LLOQ per $\mu \mathrm{L}$. The QC samples were prepared by diluting the $100 \times$ LLOQ per $\mu \mathrm{L}$ NAT peptide mix to give final concentrations of $0.4 \times(\mathrm{QC}-\mathrm{A}), 5 \times(\mathrm{QC}-\mathrm{B})$, and $50 \times(\mathrm{QC}-\mathrm{C})$ LLOQ per $\mu \mathrm{L}$. Three replicates per QC concentration were prepared and analyzed along with each batch of samples.

Solid-phase extraction and SIS addition. An aliquot consisting of $55 \mu \mathrm{L}$ of each plasma digest sample was transferred to a well in an Eppendorf LoBind skirted PCR plate, along with $55 \mu \mathrm{L}$ of the surrogate matrix (10 $\left.\mathrm{mg} \mathrm{mL}^{-1} \mathrm{BSA}\right)$ for each QC sample and standard. The SIS peptide mixture was solubilized in $220 \mu \mathrm{L}$ of $30 \% \mathrm{ACN} / 0.1 \% \mathrm{FA}$, transferred to a $15 \mathrm{~mL}$ Falcon tube, and then diluted to $10 \times$ LLOQ per $\mu \mathrm{L}$ with $0.1 \%$ FA. The eight-point standard curve was prepared by combining the surrogate matrix, SIS peptide mixture, and the level-specific light peptide mixture at a ratio of $1: 1: 1(\mathrm{v} / \mathrm{v} / \mathrm{v})$. All plasma and QC samples were spiked with the same amount of the SIS peptide mixture as the standards. Plasma samples, QC samples, and standards were then concentrated by solid-phase extraction (SPE) using an Oasis HLB $\mu$ Elution plate. Briefly, the SPE plate was conditioned with $600 \mu \mathrm{L} \mathrm{MeOH}$, equilibrated with $600 \mu \mathrm{L}$ of $0.1 \%$ aqueous $\mathrm{FA}$ followed by loading the plasma sample digests, QC samples, and standards. The wells were washed three times with $600 \mu \mathrm{L}$ of $\mathrm{H}_{2} \mathrm{O}$, and the bound peptides were eluted with $55 \mu \mathrm{L}$ of $70 \%$ ACN/0.1\% FA. After the SPE step, the concentrated eluate was evaporated using a speed vacuum concentrator and then stored at $-80{ }^{\circ} \mathrm{C}$. Plasma samples, standards and QC samples were then resolubilized and analyzed on the Agilent 6495B.

LC separation and MS analysis. Samples were solubilized with aqueous $0.1 \%$ FA to give a final peptide mix concentration of $1 \mu \mathrm{g} \mu \mathrm{L}^{-1}$ for LC/MRM-MS analysis. Of each of the rehydrated plasma digests, QCs and standards, $10 \mu \mathrm{L}$ were injected and separated with a Zorbax Eclipse Plus RP-UHPLC column $(2.1 \times 150 \mathrm{~mm}, 1.8 \mu \mathrm{m}$ particle diameter; Agilent), contained within an Agilent 1290 Infinity system and maintained at $50{ }^{\circ} \mathrm{C}$. The performance of UHPLC and nano-HPLC for MRM-based quantitation of putative plasma biomarker proteins has been previously investigated. ${ }^{30}$ UHPLC was found to be the better choice, when sufficient amount of sample can be obtained. In this case, UHPLC was found to provide overall higher sensitivity for the majority of peptides tested, and more stable retention times. Thus, for our purposes, peptide separations were achieved at UHPLC flow rates of $0.4 \mathrm{~mL} \mathrm{~min}^{-1}$ over a 60 min run, via a multi-step LC gradient. The aqueous mobile phase was composed of $0.1 \%$ FA in LC-MS grade water and the organic mobile phase of $0.1 \%$ FA in LC-MS grade ACN. The gradient was set up to start at $2 \%$ organic mobile phase, increase to $7 \%$ at $2 \mathrm{~min}$, to $30 \%$ at $50 \mathrm{~min}, 45 \%$ at $53 \mathrm{~min}$, $80 \%$ at $53.5 \mathrm{~min}$ and hold at $80 \%$ until $55.5 \mathrm{~min}$, go back to $2 \%$ at $56 \mathrm{~min}$, and then hold at $2 \%$ until $60 \mathrm{~min}$. A post-gradient column re-equilibration of $4 \mathrm{~min}$ was used after the analysis of each plasma sample, QC sample, and standard. 
MS analysis was performed on an Agilent 6495B triple quadrupole instrument operated in the positive ion mode. MRM data were acquired at $3.5 \mathrm{kV}$ and $300 \mathrm{~V}$ capillary voltage and nozzle voltage, respectively. The sheath gas flow was set to $11 \mathrm{~L} \mathrm{~min}^{-1}$ at a temperature of $250{ }^{\circ} \mathrm{C}$, and the drying gas flow was set to $15 \mathrm{~L} \mathrm{~min}^{-1}$ at a temperature of $150{ }^{\circ} \mathrm{C}$, with the nebulizer gas pressure at $30 \mathrm{psi}$. The collision cell accelerator voltage was set to $5 \mathrm{~V}$, and unit mass resolution was used in the first and third quadrupole mass analyzers. The high energy dynode (HED) multiplier was set to $-20 \mathrm{kV}$ for improved ion detection efficiency and signal-to-noise ratios. A single transition per peptide target was monitored over $700 \mathrm{~ms}$ cycles and $90 \mathrm{~s}$ detection windows were used for the quantitative analysis.

Data analysis. Skyline Quantitative Analysis software (version 19.1.0.193, University of Washington) ${ }^{31}$ was used to visually examine the resulting LC/MRM-MS data. The chromatographic peaks for the NAT and SIS peptides in the plasma samples, calibration curves and QCs were assessed manually for shape and accurate integration. Calibration curves were generated using $1 / x^{2}$-weighted linear regression and were used to calculate the peptide concentrations in the samples as fmol per $\mu \mathrm{L}$ of plasma (see Fig. 1). Further data analysis and visualization was performed using $\mathrm{R}$, including the generation of volcano plots and the heatmap with hierarchal clustering.

Standard and quality control acceptance criteria. The standards and QC samples were examined, and either accepted or rejected based on a set of rules and criteria. Standards and QC samples were acceptable if the values fell within $\pm 20 \%$ of the theoretical concentration. A standard curve was deemed to be acceptable if the back-calculated concentrations of at least 5 out of the 8 standards were found to be within $\pm 20 \%$ of the theoretical concentration at each point, including the LLOQ. Similarly, at least $66 \%$ of all QC samples were required to fall within $\pm 20 \%$ of the theoretical concentration. An experiment

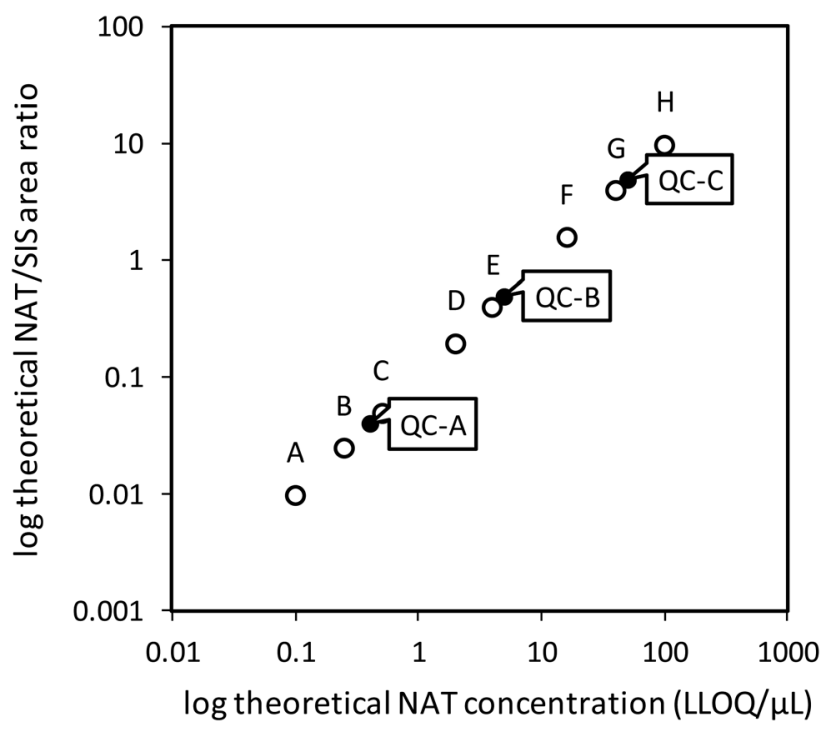

Fig. 1 Overview of theoretical concentrations for calibration curve points and quality control samples. was deemed to be successful if at least $90 \%$ of the peptide calibration curves were acceptable and passed criteria.

\section{Results and discussion}

\section{Performance of calibration curves and QCs}

Three sets of standard curves were generated to determine the precision and accuracy in the standards used for the calibration curve, the QC samples, and the 21 different plasma lots. The calibration curves and QC samples were evaluated following the acceptance criteria described in the previous section (at least 5/8 calibration curve standards, and at least $66 \%$ of all QC samples were within $\pm 20 \%$ of the theoretical concentration, i.e. the expected concentration based on our calculations). As presented in Table 1, more than $93 \%$ of all standards and QC samples acquired during the three-day study met the target criteria.

The standard curves for all 267 peptides met the criteria shown above and were within $\pm 20 \%$ of the theoretical value for a minimum of 5 out of 8 standard levels. As can be seen in Fig. 2, the majority of the three sets of standard curves were generated with 8 out of 8 standard points, followed by 7, 6, and 5 points, demonstrating the linear performance of the selected peptides for MRM analysis over several orders of magnitude and giving high confidence in the protein quantitation results.

As shown in Fig. 3, all three QC levels performed as expected and fell within acceptable limits for the majority of proteins. The few rejected QCs were either outside the linear range (OR) or outside the acceptable limits (OAL). Thus, in a curve where standards A $(1 \times$ LLOQ $)$ and B $(2.5 \times$ LLOQ $)$ were rejected, QC-A ( $4 \times$ LLOQ) was automatically rejected and denoted "OR". The lowest point on curve (LPOC), or LLOQ for that particular curve, became standard $\mathrm{C}(5 \times)$. In this case, the curve itself was deemed to be acceptable if the other two QC samples (QC-B and QC-C) passed the acceptance criteria. The OAL annotation was used for QC samples that fell outside the $\pm 20 \%$ acceptance criterion, and fewer than $1 \%$ of the QC samples were OAL. This suggests proper performance of the calibration curve and QC samples for most peptides, thus providing high confidence in the quantitative results of the plasma samples analyzed.

\section{Protein quantitation in 21 lots of commercial human plasma}

Quantitation of all 267 proteins was attempted for all 21 sample lots (10 male, 10 female, 1 pooled sample; see ESI Table $2 \dagger$ ) using the three standard curves prepared on days 1

Table 1 Overview of the percentages of curve standards and QC samples meeting acceptance criteria for analysis days 1 to 3

\begin{tabular}{lll}
\hline Day & Accepted curve standards (\%) & Accepted QC samples (\%) \\
\hline 1 & $94.4 \%$ & $95.0 \%$ \\
2 & $95.5 \%$ & $93.9 \%$ \\
3 & $96.5 \%$ & $94.1 \%$
\end{tabular}




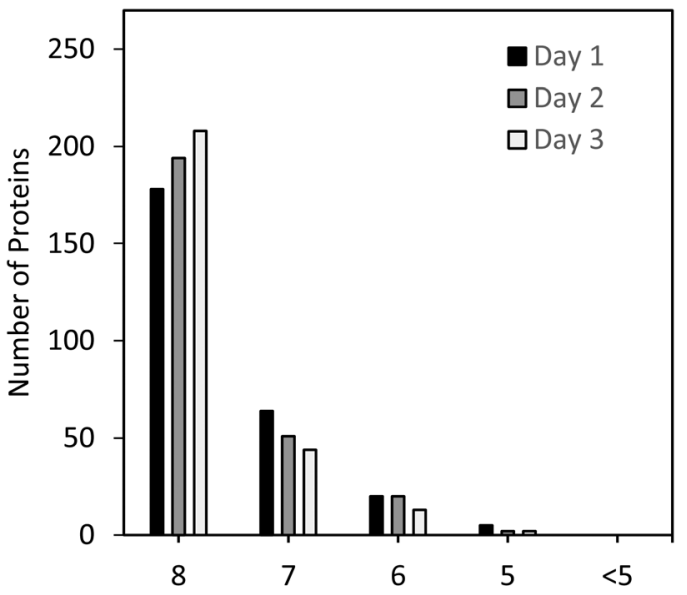

Number of standards within curve criteria

Fig. 2 Number of proteins with $8,7,6,5$, and $<5$ points (out of 8 points per standard curve) meeting the acceptance criteria for each protein measured on days 1 to 3 . A standard curve was deemed to be acceptable if the back-calculated concentrations of at least 5 out of 8 standards were found to be within $\pm 20 \%$ of the theoretical concentration at each point, including the LLOQ.

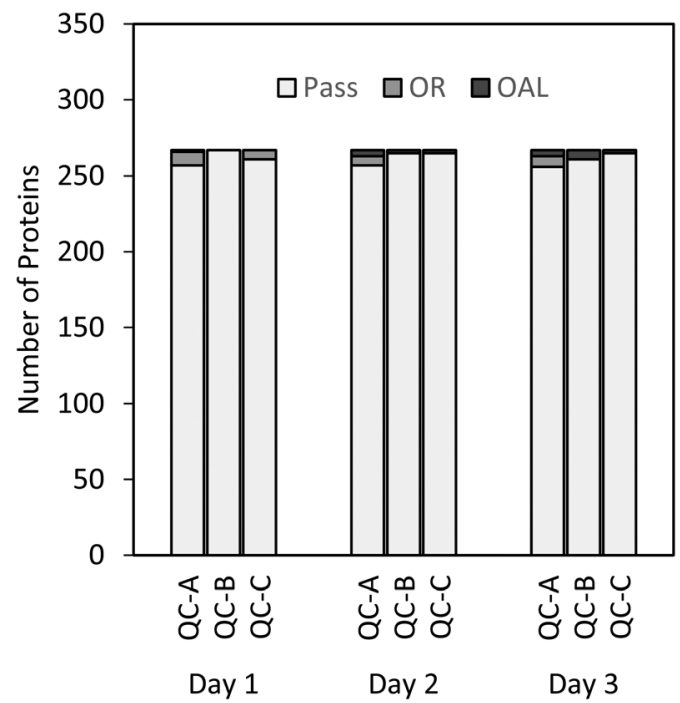

Fig. 3 Number of protein QCs meeting the criteria for each of the 267 proteins measured on days 1 to 3 . OR = out of linear range; OAL = out of acceptable limits; Pass = at least one of the three QC samples at each level was within $\pm 20 \%$ of the theoretical concentration.

to 3. All standard curves were generated with five to eight standards, as shown in Fig. 2. Each standard curve possessed an LLOQ and a ULOQ, as determined by the lowest and highest passing standards, respectively. In total, 248 proteins were quantifiable (within the linear range) in at least one sample on at least one day, and 157 proteins were quantifiable in at least one sample on all three days. A total of 111 proteins were quantifiable in all 21 samples on all three days. Notably, no protein in any sample was found to be above the ULOQ, and 110 proteins were below the LLOQ in all samples. Sixty of these proteins are thought to be associated with diseases such as neuropathy, lung cancer, epilepsy, rheumatoid arthritis, cardiomyopathy, among others, according to UniProt. ${ }^{28}$ Hence, despite not all proteins being quantifiable across all samples, their analysis is still relevant, for instance when assessing different diseases which might result in up-regulation of some of the proteins.

The number of proteins quantified between different samples and between different days of analysis was found to be very consistent (see Fig. 4), with a median of 145 proteins quantified, and a standard deviation of 15.8. Furthermore, the overlap of the quantifiable proteins per sample was considerable with on average $84 \%$ of the proteins being quantified across all three days (see ESI Fig. 1†). Interestingly, two samples (BRH1447341, day 3, and BRH1447347, day 1), showed elevated numbers of proteins quantified on one of the three days. A possible explanation for this phenomenon could be a slight heterogeneity in the sample aliquots, for example, caused by plasma clots. This is supported by the observation that the QC samples and standard curves across all three days are very similar, thus ruling out LLOQ shifts of the calibration curves between days as the cause of the elevated numbers of proteins. This highlights the importance of adhering to strict sample preparation protocols to ensure as little variation as possible.

Overall, the data demonstrated the high reproducibility of the MRM approach for highly multiplexed protein quantitation.

Fig. 5 shows the distribution of \% CVs calculated for all the proteins that were quantifiable in the plasma samples on each of the three days (a total of $2793 \mathrm{CVs}$ were calculated for the 21 samples analyzed). More than $86 \%$ of the CVs were below $15 \%$ $\mathrm{CV}$, with the majority falling below $10 \% \mathrm{CV}$. Furthermore, $6 \%$ of the CVs were between $15-20 \%$, 4.6\% were between $20-30 \%$, and only $3.4 \%$ of the CVs were $>30 \%$. This shows the quality and precision of the selected peptides and demonstrates their suitability for reliable measurements and quantitative analysis in a variety of samples, which could easily be transferred to a clinical setting.

\section{Concentration range}

Fig. 6A shows the average protein concentrations for each protein that could be quantified in three replicate analyses in at least one of the 21 plasma samples. The concentration ranges across all plasma samples were very similar, and covered approximately six orders of magnitude, from $\sim 1 \mathrm{fmol}$ $\mu \mathrm{L}^{-1}$ for P-selectin, to $747 \mathrm{pmol} \mu \mathrm{L}^{-1}$ for human serum albumin, which falls within the normal accepted concentration range for serum albumin. ${ }^{32}$ Furthermore, the concentrations for the proteins quantified in all 21 samples across all three days (111 proteins) were very comparable, ranging from $4.13 \mathrm{fmol} \mu \mathrm{L}^{-1}$ to $747 \mathrm{pmol} \mu \mathrm{L}^{-1}$. This demonstrates the suitability of MRM for quantitation of proteins that vary in concentration by many orders of magnitude.

Plasma protein concentration measurements using traditional ELISA methods versus modern LC-MS/MS techniques have been compared previously. Strong correlation was found between the 


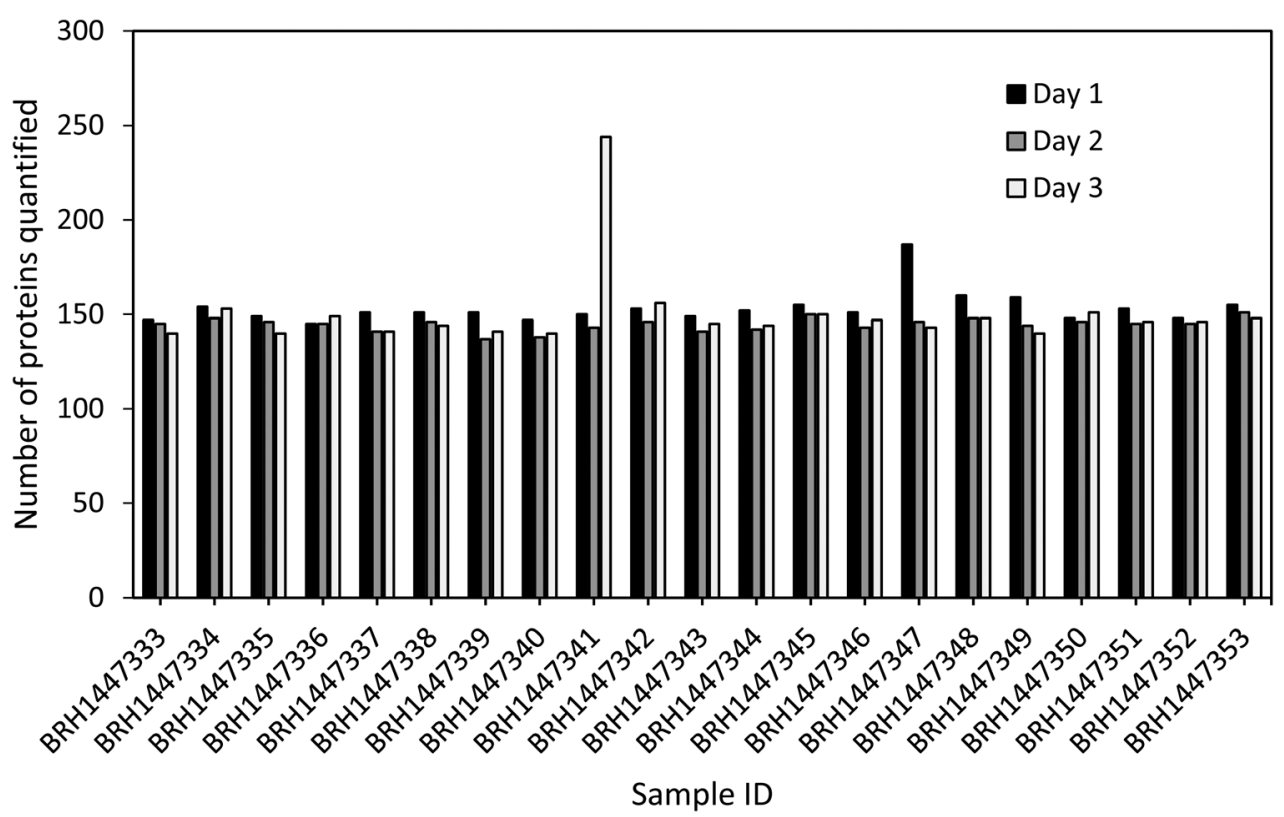

Fig. 4 Number of proteins with quantifiable endogenous concentrations as well as standard curves and QC samples within the acceptance criteria in 21 plasma samples across three days.

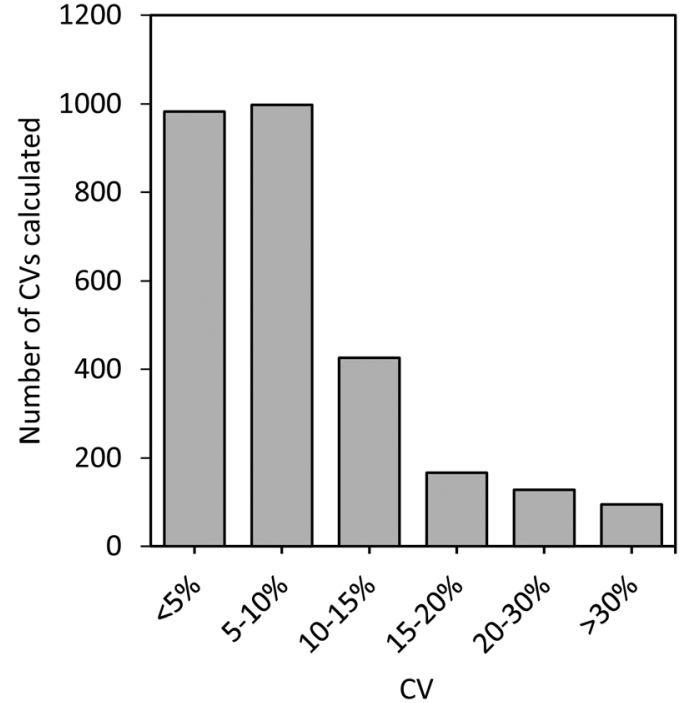

Fig. 5 Distribution of \% CVs calculated for all sample protein concentrations determined on all three days.

two methods when measuring plasma PCSK9 in 30 human plasma samples. ${ }^{33}$ Although discrepancies between ELISA and LC-MS/MS have been documented in the past, further investigation has revealed that the strong dependence of ELISAs on the antibody specificity can become a hinderance. For example, the discrepancy found between ELISA and LC-MS/MS assay results on a PEGylated scaffold protein in post-dose monkey samples was shown to be due to ELISA measuring only the active circulating drug (target-binding), whereas the LC-MS/MS method measured the total circulating drug. ${ }^{34}$ The bias observed in ELISA results supports that LC-MS/MS could in many instances reveal a more accurate picture, as reviewed previously. ${ }^{35}$ Thus, we believe that a direct comparison between ELISA and our LC-MRM-MS methods would be of limited value.

Of the 267 proteins, 144 proteins were quantified in at least 5 of the 21 samples, and differences in protein concentrations were determined (Fig. 6B). Of these 144 proteins, 48 are FDAapproved/laboratory developed test (LDT) biomarkers, as listed by Anderson et al. ${ }^{23}$ Depending on the protein, these concentration ranges were as low as 1.1-fold for metalloproteinase inhibitor 2, to 69-fold for serum amyloid A-1/A-2 (see ESI Table $3 \uparrow$ for details). The highest fold-difference observed for these FDA-approved biomarkers was 60-fold for C-reactive protein. The data show that although most protein abundances between samples are greatly conserved, some proteins show significant differences. For instance, C-reactive protein and serum amyloid $\mathrm{A}$, both recognized as acute phase proteins, are known to increase in concentration by several hundred-fold or more in response to inflammation. ${ }^{36,37}$ The differences in protein abundances between samples highlight the importance of, ideally, establishing patient-specific baseline measurements in preventative medicine and disease monitoring rather than establishing a baseline for a cohort of patients. Furthermore, it highlights the suitability of MRM-MS based methods for their multiplexing capabilities across multiple orders of magnitude.

\section{Data assessment for statistically significant differences}

The protein concentrations determined for all samples were further assessed for statistically significant differences between male and female, as well as ethnicity (Hispanic, Black and Caucasian). It is important to note that only one sample was from a Caucasian donor. 

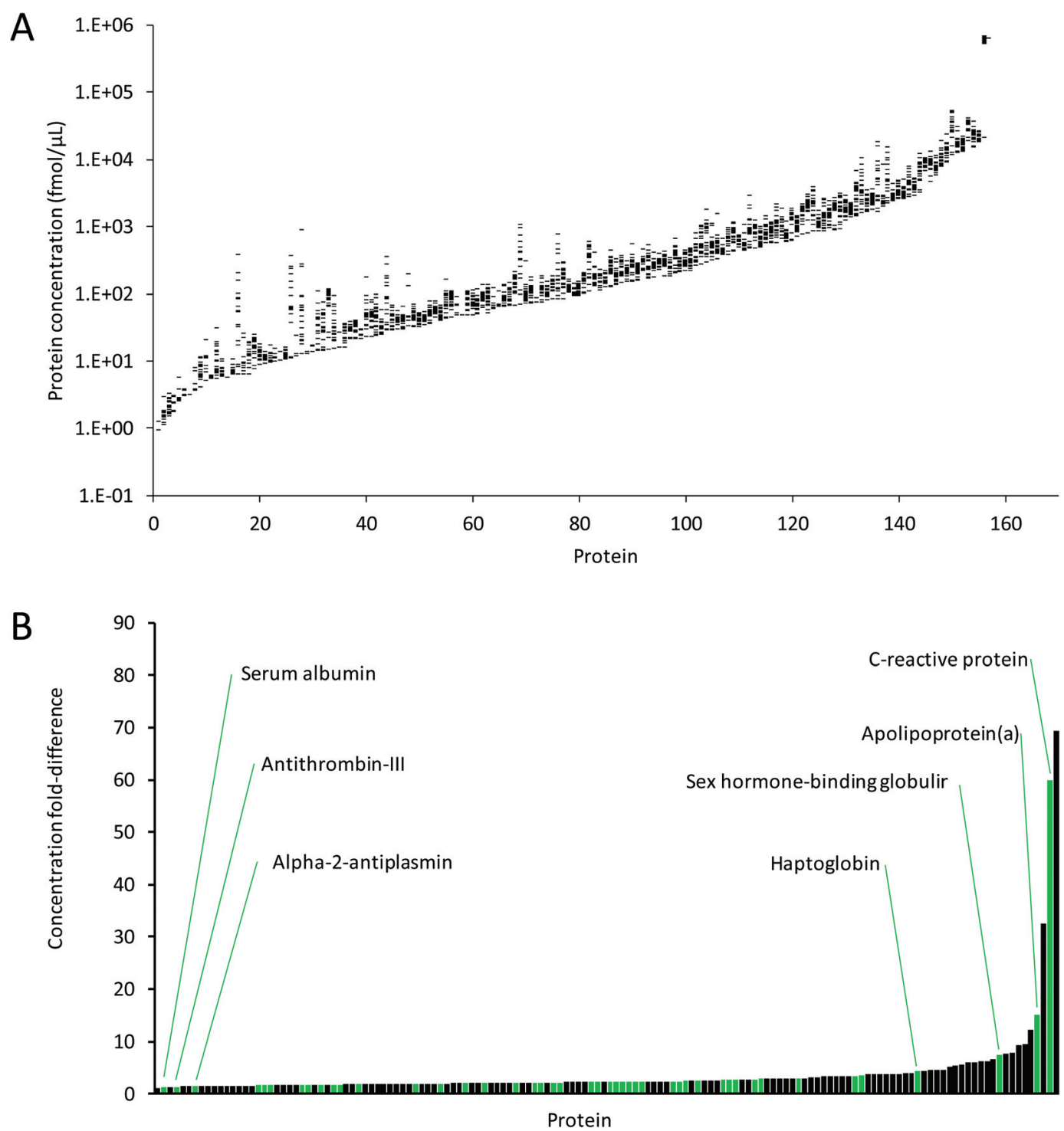

Fig. 6 (A) Ranges of average concentrations for the proteins quantified across three days in the 21 plasma samples. Proteins are sorted by minimum concentration across all samples. (B) Concentration ranges (i.e. fold-differences) between samples for proteins with quantifiable protein concentrations in at least 5 samples (144 proteins). Green bars show the FDA-approved and LDT biomarkers, as listed by Anderson et al. ${ }^{23}$

Upon statistical analysis, the concentrations for the majority of proteins showed no significant differences between Black and Hispanic groups (Fig. 7A). All but four of the values were within the \pm 0.5 -fold change. The hemoglobin subunit alpha (P69905), Apolipoprotein A-IV (P06727) and Apolipoprotein C-III (P02656) concentrations were found to be significantly higher in the Hispanic group than in the Black subject, while the immunoglobulin heavy constant gamma 1 (P01857) was significantly lower in the Hispanic group than in the Black subject.

The plasma concentrations for hemoglobin subunit alpha (P69905) were found to be comparable between female and male in the samples from Black subjects. In contrast, the samples from male subjects with a Hispanic background showed 3-fold higher average levels than the female Hispanic samples, and approximately 6-fold higher average levels than the samples from Black subjects (Fig. 7B). Furthermore, male subjects showed slightly elevated concentrations compared to female subjects in both the Hispanic and Black groups for Apolipoprotein A-IV (P06727; Fig. 7C) and Apolipoprotein C-III (P02656; Fig. 7D). Immunoglobulin heavy constant gamma 1 (P01857), however, was found to have a higher concentration in Black subjects compared to Hispanic subjects (Fig. 7E). It is important to note, however, that the spread of P01857 concentrations in all groups was wide, which reflects a natural spread of IgG and the function of the immune system. Here, as we do not have any additional information on the medical history of these samples, it is hard to reach any definitive conclusion about why P01857 is elevated in one group and not another. Nonetheless, this demonstrates the ability of the assay to 
A

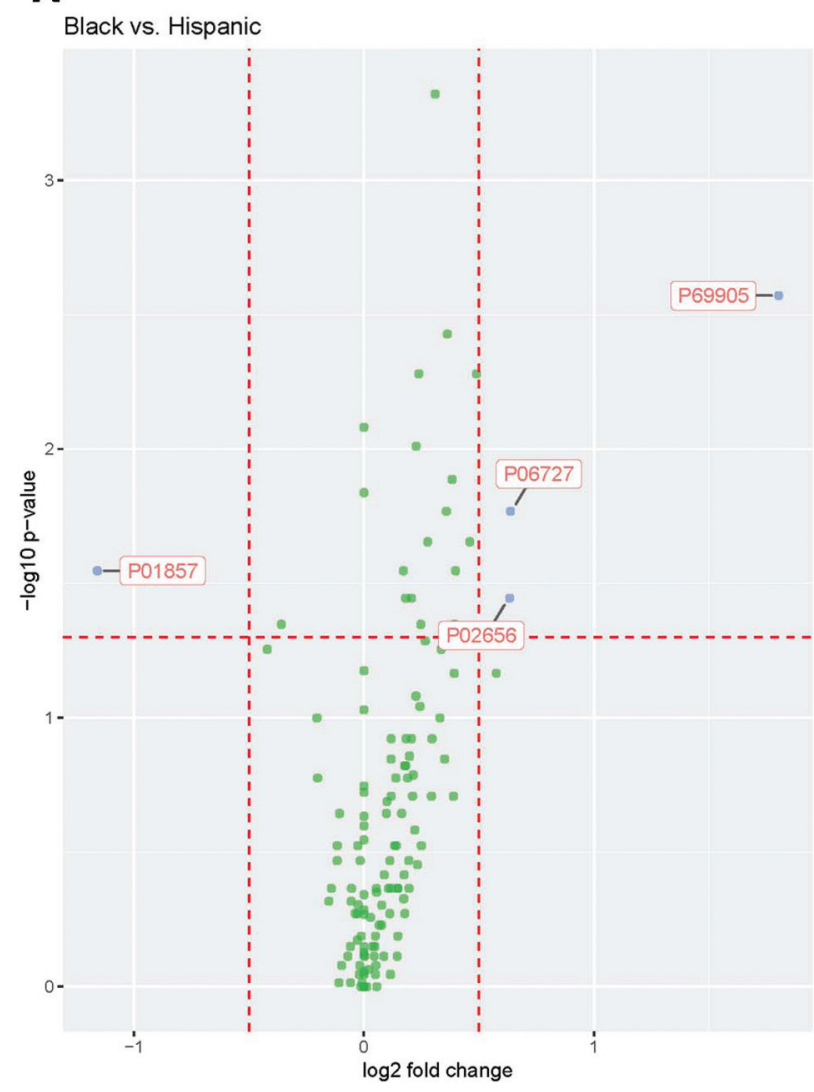

B

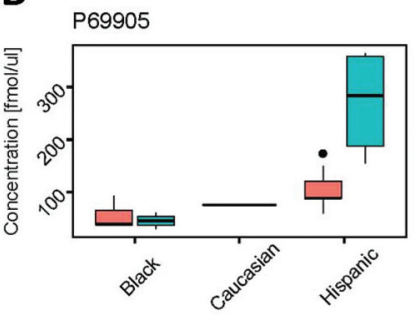

D

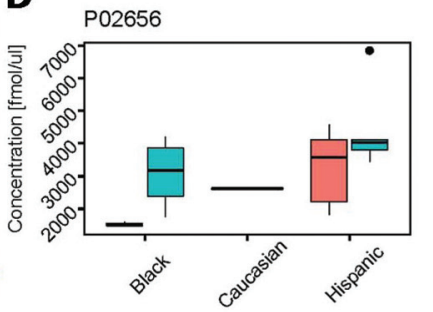

C

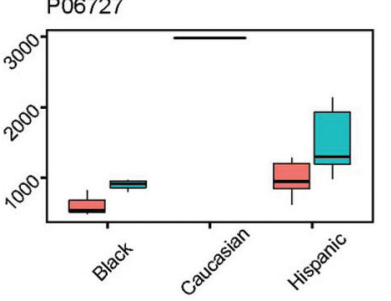

E

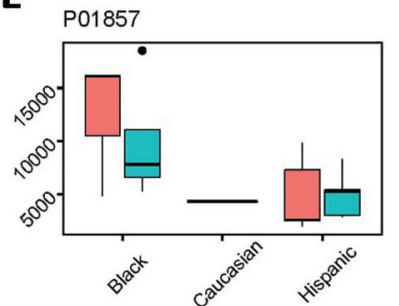

Sex

官 Female

白 Male

Fig. 7 Assessment of Black vs. Hispanic subject groups for statistical differences in proteins quantified across all plasma samples. (A) Volcano plot. (B-E) Box plots for protein concentrations determined for (B) hemoglobin subunit alpha (P69905), (C) Apolipoprotein A-IV (P06727), (D) Apolipoprotein C-III (P02656), and (E) immunoglobulin heavy constant gamma 1 (P01857).

capture and measure the elevation of immunoglobulin heavy constant gamma 1 levels, which could potentially be used to monitor the function of the immune system.

A similar trend was seen when assessing the protein concentration differences between male and female groups (Fig. 8).

While the majority of concentration values showed no statistically significant differences (Fig. 8A), the proteins CD5 antigen-like (O43866; Fig. 8B), sex hormone-binding globulin (P04278; Fig. 8C), fibronectin (P02751; Fig. 8D), and immunoglobulin heavy constant mu (P01871; Fig. 8G and H) were significantly higher in females than in males. This observation for immunoglobulin heavy constant mu agrees with previously reported data by Oyeyinka $\mathrm{et} \mathrm{al.}{ }^{38}$

Interestingly, the two different tryptic peptides (VSVFVPPR and GFPSVLR) used as surrogates to quantify immunoglobulin heavy constant mu (P01871), showed the same fold difference, demonstrating the validity of this approach to assess quantitative differences between samples. Despite the fact that both peptides were used to quantify the same protein and showed a statistically significant difference between female and male samples, the absolute concentration values between the two peptides differed by a factor of approximately 1.5, suggesting different release efficiencies and/or degradation behaviour of these peptides. ${ }^{39}$ The excellent correlation of the quantitative results (with an $R^{2}$ value of 0.997 , see ESI Fig. $2 \dagger$ ), however, indicates that the concentrations determined are highly reproducible and therefore valid for protein quantitation. Furthermore, the concentrations of the proteins Apolipoprotein A-IV (P06727; Fig. 8E) and Apolipoprotein D (P05090; Fig. 8F) were significantly lower in females than in males.

Additionally, hierarchical clustering showed no obvious clusters (Fig. 9 and ESI "Gaither_Popp_Borchers_ Figure_9_data.csv" $\dagger$ ), further supporting the lack of statistically significant differences in the majority of protein concentrations between samples, although sub-clusters for male and female within the Black and Hispanic groups were observed.

Taken together, the data suggest that there are no significant concentration differences in all of the human plasma samples analyzed, supporting the idea that determining protein concentration references ranges for groups of patients, such as patients considered "healthy", is a valid approach. Previous studies using subsets of these assays on samples from cancer and control patients demonstrated the ability to capture a protein signature reflecting the two groups. $^{40}$ 
A

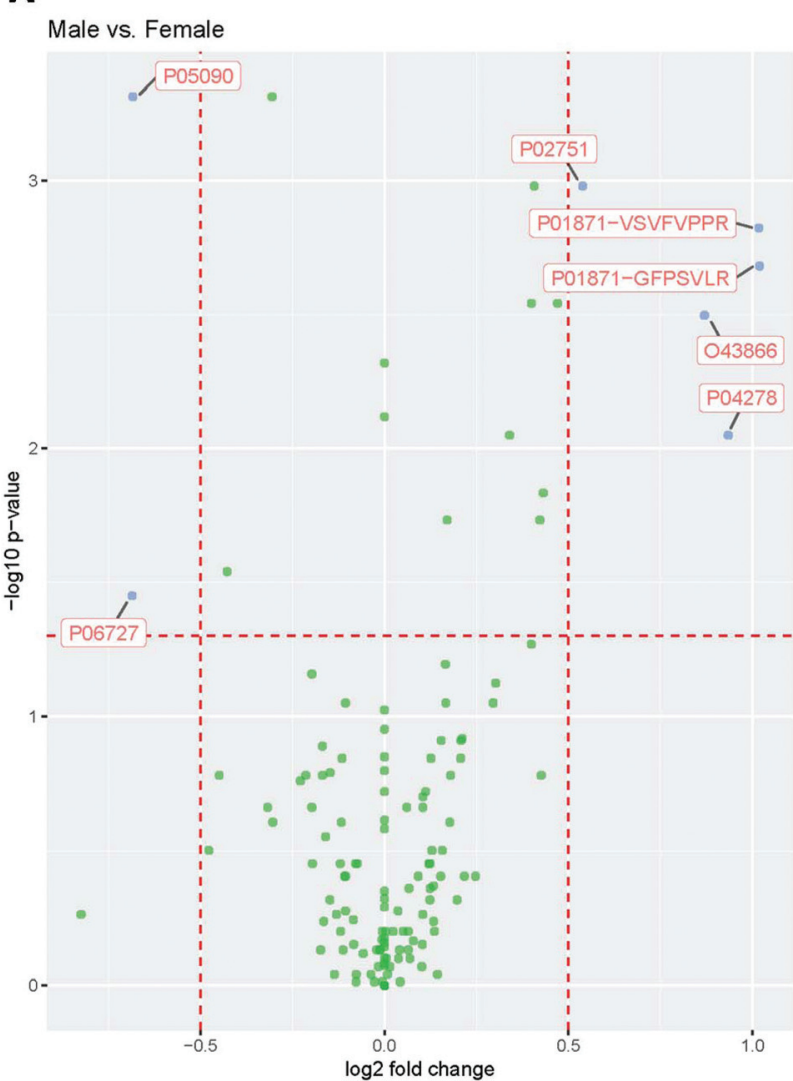

B

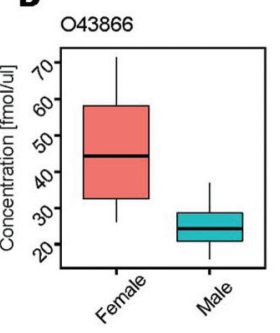

E

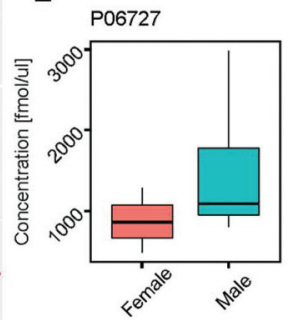

G

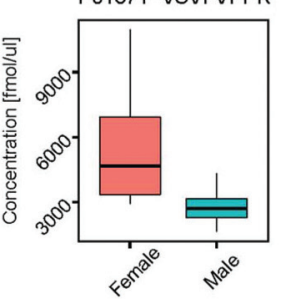

C

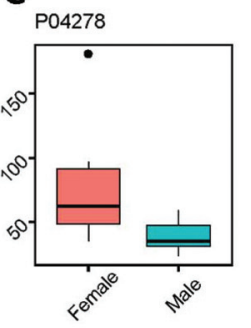

$\mathbf{F}$

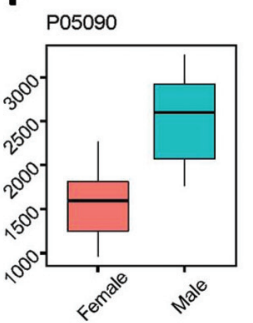

H

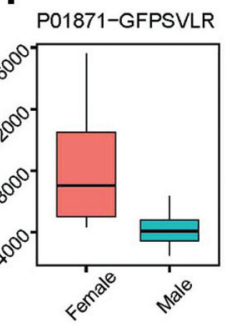

D

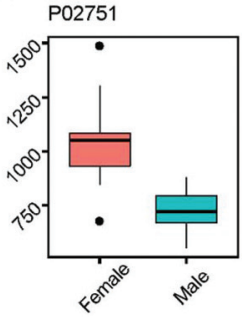

Fig. 8 Assessment of male vs. female groups for statistical differences in proteins quantified across all plasma samples. (A) Volcano plot. (B-H) Box plots for protein concentrations determined for (B) CD5 antigen-like (O43866), (C) sex hormone-binding globulin (P04278), (D) fibronectin (P02751), (E) Apolipoprotein A-IV (P06727), (F) Apolipoprotein D (P05090), and the tryptic peptides (G) GFPSVLR and (H) VSVFVPPR from immunoglobulin heavy constant mu (P01871).

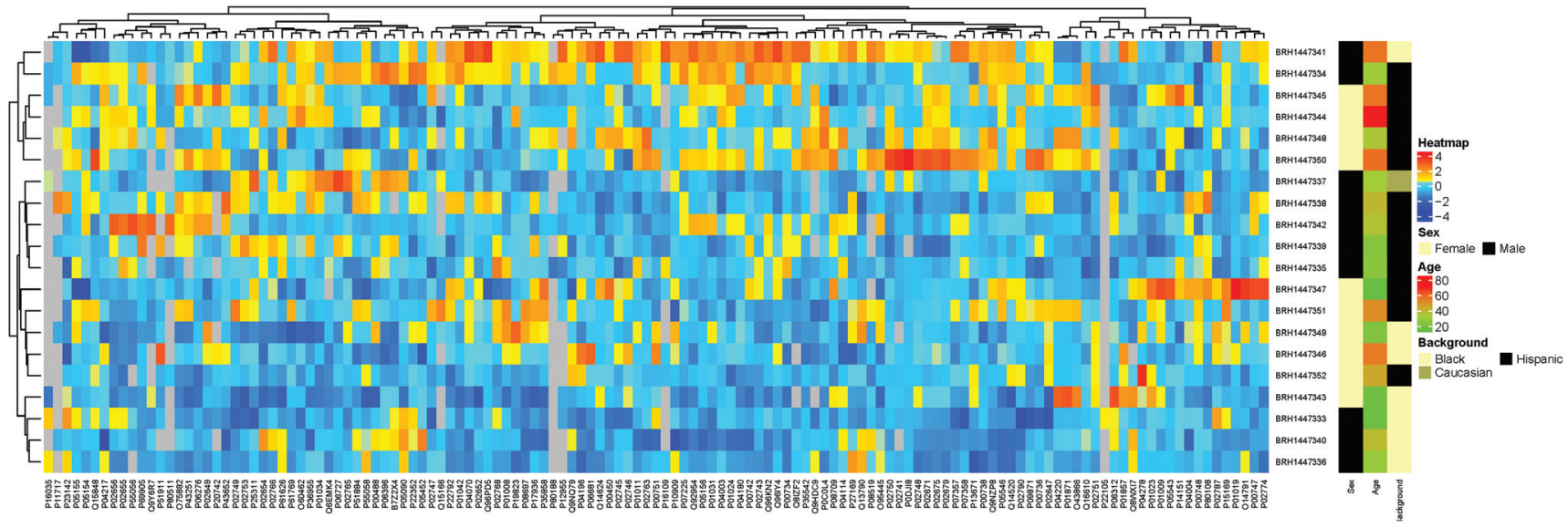

Fig. 9 Hierarchical clustering results for the patient samples analyzed, using ethnic background and sex as groups to be compared.

\section{Conclusion}

The PeptiQuant 267-protein MRM kit has been successfully used for determining the protein concentrations across six orders of magnitude in 21 commercially available human plasma samples in a highly reproducible and accurate fashion using stable isotope-labelled standard peptides. We believe this approach is well-suited to advance the biomarker discovery efforts to the next stage, biomarker validation, to finally bring more biomarkers into the clinic. Additionally, the poten- 
tial for differentiating diseased state $v s$. healthy state with peptide surrogates with mutated sequences as observed in a diseased state remains a possibility for the future of MRMbased clinical diagnostics and a project we look forward to exploring in the future.

\section{Conflicts of interest}

CHB is the CSO of MRM Proteomics, Inc. CG and RP are staff members of MRM Proteomics, Inc.

\section{Acknowledgements}

MRM Proteomics Inc. is grateful for the efforts of our collaborators at the team at the University of Victoria - Genome BC Proteomics Centre, in particular Andrea Palmer and Derek Smith, in synthesizing, characterizing, and validating the 267 MRM assays. CHB and the University of Victoria-Genome British Columbia Proteomics Centre are grateful to Genome Canada and Genome British Columbia for financial support through the Genomics Innovation Network (project codes 204PRO for operations and 214PRO for technology development) and the Genomics Technology Platform (264PRO). CHB is also grateful for support from the Leading Edge Endowment Fund, and the Segal McGill Chair in Molecular Oncology at McGill University (Montreal, Quebec, Canada). CHB is also grateful for support from the Warren Y. Soper Charitable Trust and the Alvin Segal Family Foundation to the Jewish General Hospital (Montreal, Quebec, Canada).

\section{References}

1 R. Aebersold and M. Mann, Nature, 2016, 537, 347-355.

2 T. G. Cross and M. P. Hornshaw, J. Appl. Bioanal., 2016, 2, 108-116.

3 C. A. Morales-Betanzos, H. Lee, P. I. Gonzalez Ericsson,

J. M. Balko, D. B. Johnson, L. J. Zimmerman and D. C. Liebler, Mol. Cell. Proteomics, 2017, 16, 1705-1717.

4 A. N. Hoofnagle and M. H. Wener, J. Immunol. Methods, 2009, 347, 3-11.

5 A. N. Hoofnagle, J. O. Becker, M. H. Wener and J. W. Heinecke, Clin. Chem., 2008, 54, 1796-1804.

6 M. H. Elliott, D. S. Smith, C. E. Parker and C. Borchers, J. Mass Spectrom., 2009, 44, 1637-1660.

7 C. E. Parker, T. W. Pearson, N. L. Anderson and C. H. Borchers, Analyst, 2010, 135, 1830-1838.

8 M. A. Kuzyk, D. Smith, J. C. Yang, T. J. Cross, A. M. Jackson, D. B. Hardie, N. L. Anderson and C. H. Borchers, Mol. Cell. Biochem., 2009, 8, 1860-1877.

9 A. J. Percy, S. A. Michaud, A. Jardim, N. J. Sinclair, S. P. Zhang, Y. Mohammed, A. L. Palmer, D. B. Hardie, J. C. Yang, A. M. LeBlanc and C. H. Borchers, Proteomics, 2017, 17, DOI: 10.1002/pmic.201600097.
10 S. A. Michaud, N. J. Sinclair, H. Petrosova, A. L. Palmer, A. J. Pistawka, S. Zhang, D. B. Hardie, Y. Mohammed, A. Eshghi, V. R. Richard, A. Sickmann and C. H. Borchers, Commun. Biol., 2018, 1, 78.

11 A. G. Chambers, A. J. Percy, J. Yang and C. H. Borchers, Mol. Cell. Proteomics, 2015, 14, 3094-3104.

12 E. L. de Graaf, J. Kaplon, S. Mohammed, L. A. M. Vereijken, D. P. Duarte, L. R. Gallego, A. J. R. Heck, D. S. Peeper and A. F. M. Altelaar, J. Proteome Res., 2015, 14, 2906-2914.

13 Y. J. Kim, Z. Zaidi-Ainouch, S. Gallien and B. Domon, Nat. Protoc., 2012, 7, 859-871.

14 D. Domanski, L. C. Murphy and C. H. Borchers, Anal. Chem., 2010, 82, 5610-5620.

15 H. Zhang, Z. Wang, J. Stupak, O. Ghribi, J. D. Geiger, Q. Y. Liu and J. Li, Proteomics, 2012, 12, 2510-2522.

16 R. M. Schoenherr, R. G. Saul, J. R. Whiteaker, P. Yan, G. R. Whiteley and A. G. Paulovich, Mol. Cell. Proteomics, 2015, 14, 382-398.

17 M. Razavi, N. L. Anderson, M. E. Pope, R. Yip and T. W. Pearson, New Biotechnol., 2016, 33, 494-502.

18 T. A. Addona, S. E. Abbatiello, B. Schilling, S. J. Skates, D. R. Mani, D. M. Bunk, C. H. Spiegelman, L. J. Zimmerman, A.-J. L. Ham, H. Keshishian, S. C. Hall, S. Allen, R. K. Blackman, C. H. Borchers, C. Buck, H. L. Cardasis, M. P. Cusack, N. G. Dodder, B. W. Gibson, J. M. Held, T. Hiltke, A. Jackson, E. B. Johansen, C. R. Kinsinger, J. Li, M. Mesri, T. A. Neubert, R. K. Niles, T. C. Pulsipher, D. Ransohoff, H. Rodriguez, P. A. Rudnick, D. Smith, D. L. Tabb, T. J. Tegeler, A. M. Variyath, L. J. VegaMontoto, A. Wahlander, S. Waldemarson, M. Wang, J. R. Whiteaker, L. Zhao, N. L. Anderson, S. J. Fisher, D. C. Liebler, A. G. Paulovich, F. E. Regnier, P. Tempst and S. A. Carr, Nat. Biotechnol., 2009, 27, 633-641.

19 S. E. Abbatiello, B. Schilling, D. R. Mani, L. J. Zimmerman, S. C. Hall, B. MacLean, M. Albertolle, S. Allen, M. W. Burgess, M. P. Cusack, M. Ghosh, V. Hedrick, J. M. Held, H. D. Inerowicz, A. Jackson, H. Keshishian, C. R. Kinsinger, J. Lyssand, L. Makowski, M. Mesri, H. Rodriguez, P. Rudnick, P. Sadowski, N. Sedransk, K. Shaddox, S. J. Skates, E. Kuhn, D. Smith, J. R. Whiteaker, C. Whitwell, S. Zhang, C. H. Borchers, S. J. Fisher, B. W. Gibson, D. C. Liebler, M. J. MacCoss, T. A. Neubert, A. G. Paulovich, F. E. Regnier, P. Tempst and S. A. Carr, Mol. Cell. Proteomics, 2015, 14, 2357-2374.

20 A. J. Percy, J. Tamura-Wells, J. P. Albar, K. Aloria, A. Amirkhani, G. D. T. Araujo, J. M. Arizmendi, F. J. Blanco, F. Canals, J.-Y. Cho, N. Colomé-Calls, F. J. Corrales, G. Domont, G. Espadas, P. Fernandez-Puente, C. Gil, P. A. Haynes, M. L. Hernáez, J. Y. Kim, A. Kopylov, M. Marcilla, M. J. McKay, M. Mirzaei, M. P. Molloy, L. B. Ohlund, Y.-K. Paik, A. Paradela, M. Raftery, E. Sabidó, L. Sleno, D. Wilffert, J. C. Wolters, J. S. Yoo, V. Zgoda, C. E. Parker and C. H. Borchers, EuPa Open Proteomics, 2015, 8, 6-15.

21 A. J. Percy, Y. Mohammed, J. C. Yang and C. H. Borchers, Bioanalysis, 2015, 7, 2991-3004. 
22 CPTAC, https://proteomics.cancer.gov/assay-portal/about/ assay-characterization-guidance-documents, Editon edn., vol. 2015.

23 N. L. Anderson, Clin. Chem., 2010, 56, 177-185.

24 D. Domanski, A. J. Percy, J. Yang and A. G. Chambers, Proteomics, 2012, 12, 1222-1243.

25 A. LeBlanc, S. A. Michaud, A. J. Percy, D. B. Hardie, J. Yang, N. J. Sinclair, J. I. Proudfoot, A. Pistawka, D. S. Smith and C. H. Borchers, J. Proteome Res., 2017, 16, 25272536.

26 M. A. Kuzyk, C. E. Parker, D. Domanski and C. H. Borchers, Methods Mol. Biol., 2013, 1023, 53-82.

27 Y. Mohammed, D. Domański, A. M. Jackson, D. S. Smith, A. M. Deelder, M. Palmblad and C. H. Borchers, J. Proteomics, 2014, 106, 151-161.

28 The_UniProt_Consortium, Nucleic Acids Res., 2015, 43, D204-D212.

29 CPTAC_Assay_Portal, https://assays.cancer.gov, Editon edn., vol. 2015.

30 A. J. Percy, A. G. Chambers, J. Yang, D. Domanski and C. H. Borchers, Anal. Bioanal. Chem., 2012, 404, 1089-1101.

31 B. MacLean, D. M. Tomazela, N. Shulman, M. Chambers, G. L. Finney, B. Frewen, R. Kern, D. L. Tabb, D. C. Liebler and M. J. MacCoss, Bioinformatics, 2010, 26, 966-968.

32 G. Weaving, G. F. Batstone and R. G. Jones, Ann. Clin. Biochem., 2016, 53, 106-111.
33 M. Croyal, F. Fall, M. Krempf, A. Thédrez, K. Ouguerram, V. Ferchaud-Roucher, A. Aguesse, S. Billon-Crossouard, P. Mata, R. Alonso, G. Lambert and E. Nobécourt, J. Chromatogr. B: Anal. Technol. Biomed. Life Sci., 2017, 1044-1045, 24-29.

34 S. J. Wang, S. T. Wu, J. Gokemeijer, A. Fura, M. Krishna, P. Morin, G. Chen, K. Price, D. Wang-Iverson, T. Olah, R. Weiner, A. Tymiak and M. Jemal, Anal. Bioanal. Chem., 2012, 402, 1229-1239.

35 A. N. Hoofnagle and M. H. Wener, J. Immunol. Methods, 2009, 347, 3-11.

36 C. C. Yue, J. Muller-Greven, P. Dailey, G. Lozanski, V. Anderson and S. Macintyre, J. Biol. Chem., 1996, 271, 22245-22250.

37 M. De Buck, M. Gouwy, J. M. Wang, J. Van Snick, G. Opdenakker, S. Struyf and J. Van Damme, Curr. Med. Chem., 2016, 23, 1725-1755.

38 G. O. Oyeyinka, L. S. Salimonu, A. I. Williams, A. O. Johnson, O. A. Ladipo and B. O. Osunkoya, Afr. J. Med. Med. Sci., 1984, 13, 169-176.

39 J. L. Proc, M. A. Kuzyk, D. B. Hardie, J. Yang, D. S. Smith, A. M. Jackson, C. E. Parker and C. H. Borchers, J. Proteome Res., 2010, 9, 5422-5437.

40 Y. Mohammed, B. J. van Vlijmen, J. Yang, A. J. Percy, M. Palmblad, C. H. Borchers and F. R. Rosendaal, Blood Adv., 2017, 1, 1080-1087. 